\title{
Astigmatism of Skew Pencils in Optical Systems Containing Toric Surfaces
}

\section{Walter Weinstein ${ }^{2}$}

\begin{abstract}
A method is described for tracing skew rays through toric refracting surfaces and for determining the astigmatism of pencils around these rays.
\end{abstract}

\section{Introduction}

Toric refracting surfaces are used chiefly in two classes of optical systems, spectacle lenses, and anamorphotic photographic and projection objectives. In spectacles the toric surfaces are arranged to correct the axial astigmatism of the eye, and in anamorphotic systems two or more toric (possibly cylindrical) surfaces are included to give stigmatic imagery with different magnifications in different directions in the image plane.

When the axial pencil is refracted by a toric, ${ }^{3}$ some astigmatism is introduced; if a pencil is incident obliquely, either centrally or eccentrically, astigmatism of different magnitude is introduced. It is therefore desirable in the case of both types of optical systems mentioned above to determine the astigmatism of obliquely incident pencils, in order to assess the imagery in the outer parts of the field. This is the problem which was studied in the work to be described.

The problem arose in connection with spectacle lenses and may be outlined as follows:

Figure 1 shows front and side views of a spectacle lens with toric surface; $O$ is the center of the lens, $C$ is the center of rotation of the eye, and $P_{1}, P_{2}, P_{3}$ are points of incidence of the chief rays of oblique pencils. Let the meridian and equatorial sections of the toric be, respectively, in the vertical and horizontal planes $O P_{1}$ and $O P_{3}$. Then the problem for chief rays passing through the axis and through $P_{1}$ and $P_{3}$ is trivial. The chief ray itself can be traced by the ordinary ray-tracing formulas for meridian rays and spherical surfaces, and the astigmatism can be determined by the usual methods for close sagittal and tangential fans, with the slight difference that the curvatures of the refracting surface in the two directions are not equal (see eq (8)).

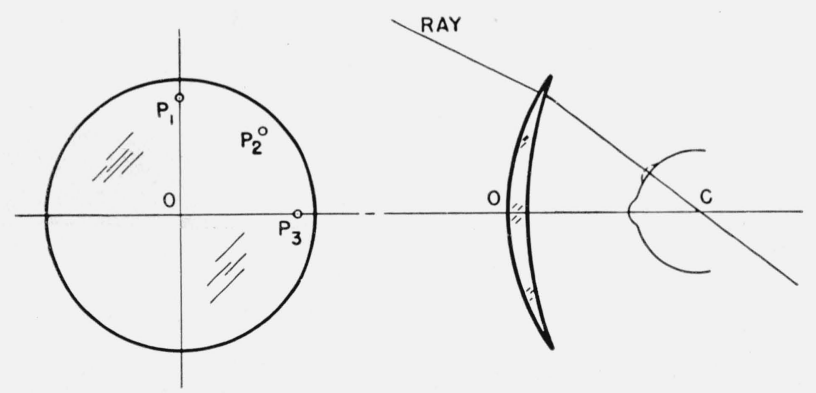

FIGURE 1.

If, however, the point of incidence of the chief ray does not lie in the meridian or equatorial section, as, for example, $P_{2}$ in figure 1 , the problem becomes more complicated. In the first place, even if the incident chief ray intersects the axis of the system, the refracted ray in general does not, so that the chief ray must for complete generality be considered to be skew; thus the first part of the problem is to trace a skew chief ray through the toric.

The work described in this paper was carried out while the author was a guest worker in the Optical Instruments Section, National Bureau of Standards, from January to July 1954.

? Present address: Imperial College, London, England.

${ }^{3}$ In what follows, "toric surface" will be abbreviated to "toric." 
Second, with oblique incidence as at any of the points $P_{1}, P_{2}$, or $P_{3}$, astigmatism is produced in the refracted pencil for two reasons: (a) because the angle of incidence is not zero, as in the ordinary case of spherical surfaces, and (b) because the refracting surface has two different principal curvatures at the point of incidence. In the case of $P_{2}$ the principal directions of the astigmatism due to (a) are parallel and normal to the plane of incidence of the chief ray, i. e., approximately parallel and perpendicular to line, $O P_{2}$; the principal directions of the astigmatism due to (b) are, however, approximately parallel to $O P_{1}$ and $O P_{3}$. Thus the resultant astigmatic pencil will have its focal lines in some intermediate directions, and the determination of these directions further complicates the calculation. ${ }^{4}$ It follows that the formal distinction between sagittal and tangential directions can no longer be made; however, for the configurations of chief ray and toric that occur in spectacle lenses the rotation of the focal lines is unlikely to be above $10^{\circ}$, so that there is little difficulty in relating the results for skew chief rays to those for rays in either principal section.

The results of such calculations would constitute a survey of the astigmatism of a toric spectacle lens at all obliquities and azimuths. They would help to answer questions such as the following: What is the best bending of a toric spectacle lens? What range of powers can be manufactured with a common back curvature without departing appreciably from the best bending? Is there any advantage in making both surfaces of a spectacle lens toric in shape? Such questions as these arise in the design of so-called best-form spectacle lenses. These have previously been designed on the basis of computations in the two principal sections only. ${ }^{5} 6$ However, an illustration is given by Henker ${ }^{6}$ showing the quality of an image produced by a pencil outside the principal sections of a typical sphero-toric lens of the Punktal best-form series; this illustration suggests that there is still room for improvement in the image. The work to be described makes it possible to compute the imagery by skew pencils and so determine whether any improvements can in fact be made.

Section 2 is a recapitulation of the properties of astigmatic pencils that are required in deriving the astigmatism equations. In section 3 the equations of a toric are given, and other properties are derived, in section 4 the ray-tracing equations for the skew chief ray are derived, and in section 5 the astigmatism formulas are derived. Section 6 deals with the forms in which the input data must be given. In section 7 the mode of presentation of the results is discussed, the results of a numerical example are given, and the possible uses of the computation are discussed.

\section{Astigmatic Pencils}

For the purposes of the present paper a pencil will be considered to be made up of a narrow bundle of rays originating from a point in the object and surrounding a chief, or principal, ray, together with all the corresponding wave fronts. The wave fronts are surfaces of constant optical path length from the object point, and the rays are normals to the wave fronts.

With a rectangular coordinate system with the $x$ axis along the principal ray, the equation of the wave front passing through the origin will take the form

$$
x=c_{20} y^{2}+c_{11} y z+c_{02} z^{2}+\text { terms of higher degree in } y \text { and } z ;
$$

linear terms are absent because the $y z$ plane must be tangent to the wave front at the origin. Also it is possible to rotate the $y$ and $z$ axes about the $x$ axis, so that the coefficient of the term in $y z$ vanishes when the equation takes the form

$$
x=\frac{1}{2}\left(c_{r} y^{2}+c_{s} z^{2}\right)+\text { higher-degree terms. }
$$

${ }_{4}^{4}$ This difficulty does not arise for points on the principal sections of the toric, such as $P_{\text {: }}$ and $P_{3}$, because then the directions of the focal lines are known by symmetry to be parallel and perpendicular to the principal sections.

${ }^{5}$ H. H. Emsley and W. Swaine, Ophthalmic lenses, 6th ed. (London, 1951).

${ }^{6} \mathrm{O}$. Henker, Introduction to the theory of spectacles (Translated by R. Kanthack) (Jena, 1924). 
Effects depending on terms in $y$ and $z$ of higher degree than the second will be neglected. To this degree of approximation it is easily shown that all rays of the pencil; i. e., normals to the surface (2) intersect one line through the point $\left(1 / c_{\tau}, 0,0\right)$ parallel to the $z$ axis and another line through the point $\left(1 / c_{s}, 0,0\right)$ parallel to the $y$ axis, these being the two astigmatic focal lines. Thus this approximation takes account of astigmatism but not of aberrations such as spherical aberration and coma, which depend on higher powers of the aperture of the pencil. The coefficients have the significance that $c_{r}$ is the curvature of the section of the wave front by the $x y$ plane and $c_{s}$, that by the $x z$ plane

Some results from differential geometry are now required. At any nonsingular point $P$ of a surface the normal curvature (i. e., the curvature of the section of the surface by a plane through the normal) has a maximum and a minimum for two section planes which are at right angles to each other; furthermore, if these two planes, together with the tangent plane at $P$, are taken as coordinate planes, the equation of the surface in the neighborhood of $P$ takes the form of eq (2), that is, the $y z$ term is absent. The maximum and minimum normal curvatures are called the principal curvatures, and the corresponding section planes meet the tangent plane at $P$ in lines that lie along the principal directions at $P$. Thus the principal directions are along the $y$ and $z$ axes for eq $(2)$.

It is also known, by Euler's theorem, that if a normal section plane makes an angle $\alpha$ with the $x y$ plane, the curvature in this section is

$$
c(\alpha)=c_{r} \cos ^{2} \alpha+c_{s} \sin ^{2} \alpha .
$$

From this it follows that these quantities are required to characterize an astigmatic pencil:

(a) The direction of the chief ray; this will be given by a unit vector, $\boldsymbol{Q}$, with components $X, Y$, and $Z$.

(b) The principal curvatures, $c_{r}$ and $c_{s}$, of the wave front at a point on the chief ray.

(c) The principal directions on the wave front; these will be given by unit vectors $\boldsymbol{R}$ and $\boldsymbol{S}$, and from the above it follows that $\boldsymbol{Q}, \boldsymbol{R}$, and $\boldsymbol{S}$ are all perpendicular to each other.

\section{Toric Surface}

A toric surface is generated by the rotation of a circle (the generating circle, abbreviated to G. C.) about an axis in its plane but not passing through its center, as in figure 2, a and b. In optical applications the lens surface is, of course, only a cap-shaped portion of the whole surface, roughly centered on the point A. Such a surface is alined with a centered system of spherical surfaces by making the optical axis lie in the normal plane to the rotation axis, which contains the center of the G. C. The optical axis then meets the toric in the pole A.

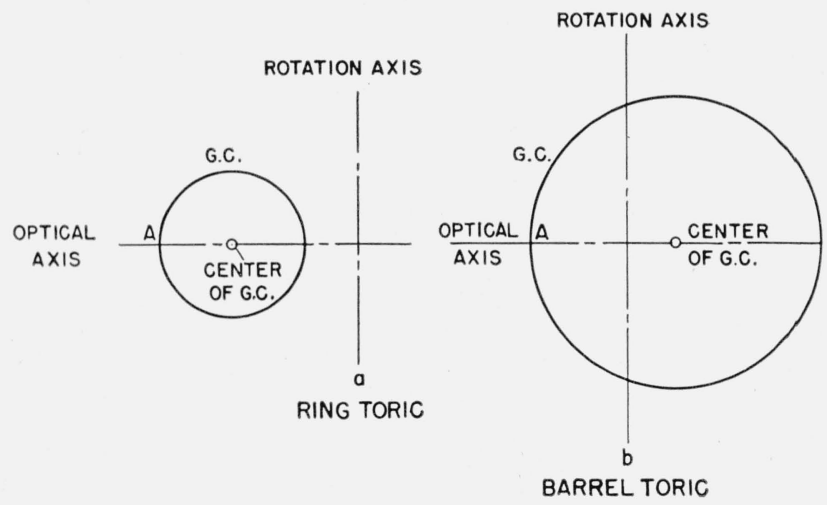

Figure 2. 
The toric refracting surface has different powers (in the optical sense) in different sections through the optical axis: the powers are determined by the radius of the $\mathrm{G}$. C. for the section in the plane of the rotation axis and by the distance from $\mathrm{A}$ to the rotation axis for that perpendicular to it; these are known as the meridian and equatorial sections, respectively.

It can be seen that for given powers in the principal sections there are two possible forms of toric surface: the radius of the G. C. can be either less than or greater than the distance from $\mathrm{A}$ to the rotation axis, the two forms being known, respectively, as ring- and barrelshaped torics. The ring form seems to be used almost exclusively for spectacles in commercial practice, but to the best of the author's knowledge, this is merely because more rings than barrels of given curvatures can be surfaced in one operation, not for any optical advantage. The possibility of improvements from the use of the barrel shape seems not to be considered in the literature on "best-form" series.

\subsection{Equations of the Toric}

One can write down the equation of the toric surface by taking rectangular coordinate axes with the origin at the pole $\mathrm{A}$ of the toric refracting surface (fig. 3 ), the $x$ axis along the optical axis, the $y$ axis parallel to the rotation axis, and the $z$ axis in the equatorial plane. Let $r_{y}$ and $r_{z}$ be the radii of curvature in the $x y$ and $x z$ sections, respectively. Take angular parameters, $\theta$ and $\phi$, as in the figure, so that $\theta$ is the longitude and $\phi$ the latitude of a point on the toric. Then the parametric equations of the toric are

$$
\left.\begin{array}{l}
x=r_{z}-\left(r_{z}-r_{y}+r_{y} \cos \phi\right) \cos \theta \\
y=r_{y /} \sin \phi \\
z=\left(r_{z}-r_{y}+r_{y} \cos \phi\right) \sin \theta
\end{array}\right\} .
$$

It can be seen that eq (4) represent a ring- or a barrel-shaped toric according as $r_{y}$ is less. than or greater than $r_{z}$ in absolute magnitude, ${ }^{7}$ in both cases the rotation axis is parallel to the $y$ axis. When the rotation axis is to be parallel to the $z$ axis the equations are obtained be interchanging $y$ and $z$ throughout, including subscripts, in eq (4); this is true also for the equations in the remainder of section 3 .

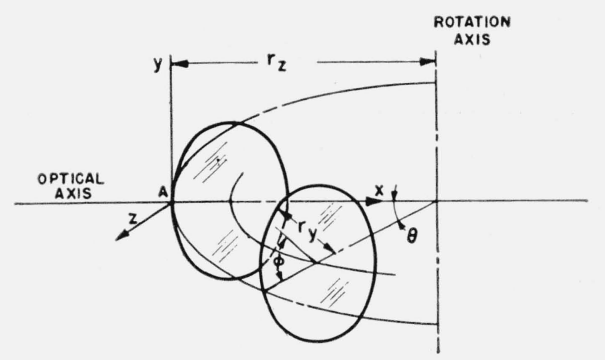

Figure 3.

An explicit equation can be obtained by eliminating $\theta$ and $\phi$ in eq (4) and solving for $x$ :

$$
x=r_{z} \pm\left\{\left[r_{z}-r_{y} \pm\left(r_{y}{ }^{2}-y^{2}\right)^{1 / 2}\right]^{2}-z^{2}\right\}^{1 / 2} .
$$

The ambiguous signs are due to the fact that there are four (possibly complex) values of $x$ for any given $y$ and $z$, since the toric is a quartic surface; the required solution is that which tends to zero as $y$ and $z$ tend to zero, so that the correct signs are, respectively, minus and plus. When these are inserted the result is unsuitable for numerical computation when $y$ and

\footnotetext{
${ }^{7}$ Provided $r_{y}$ and $r_{z}$ have the same sign, which is generally the case in spectacle lenses.
} 
$z$ are small compared to $r_{y}$ and $r_{z}$, as is generally the case. This difficulty can be circumvented by transforming the equation by means of conjugate surds in the usual way and using curvatures instead of radii, giving

where

$$
x=\frac{F}{G\left\{G+\left[G^{2}-c_{z} F\right]^{1 / 2}\right\}},
$$

$$
F=c_{z} z^{2} G^{2}+2 c_{y} y^{2} G-c_{y}{ }^{2} c_{z} y^{4}, G=1+\left(1-c_{y}{ }^{2} y^{2}\right)^{1 / 2} .
$$

In this form the equation of the toric is suitable for numerical computation with all relevant ranges of the variables.

\subsection{Principal Directions and Curvatures}

In considering the refraction of an astigmatic pencil through a toric, it is necessary to know the shape of the toric in the neighborhood of the point of incidence. Thus, as in section 2, the following are required:

(a) The direction of the normal at the point of incidence; this will be given by a unit vector, $\boldsymbol{U}$, with components, $\lambda, \mu$, and $\nu$.

(b) The principal curvatures, $c_{v}$ and $c_{w}$, at the point of incidence.

(c) The principal directions at the point of incidence; these will be specified by unit vectors, $\boldsymbol{V}$ and $\boldsymbol{W}$, and again the vectors, $\boldsymbol{U}, \boldsymbol{V}$, and $\boldsymbol{W}$, form a trirectangular system. These quantities are easily determined for a current point $(x, y, z)$; if the toric has principal curvatures, $c_{y}$ and $c_{z}$, at the origin and if the rotation axis is parallel to the $y$ axis, as in section 2.1 , the results are

$$
\begin{gathered}
U=(\cos \phi \cos \theta,-\sin \phi,-\cos \phi \sin \theta), \\
\phi=\arcsin c_{y} y, \theta=\arcsin \frac{G c_{z} z}{G-c_{z} c_{y} y^{2}}, \\
c_{v}=c_{y} \\
\left.c_{w}=\frac{G c_{z} \cos \phi}{G-c_{y} c_{z} y^{2}}\right\}, \\
\left.\begin{array}{r}
V=(\sin \phi \cos \theta, \cos \phi,-\sin \phi \sin \theta) \\
W=(\sin \theta, \quad 0, \quad \cos \theta)
\end{array}\right\} .
\end{gathered}
$$

\section{Tracing the Skew Chief Ray}

Using the quantities defined in sections 2 and 3.2, the problem can be stated as follows:

Given $\boldsymbol{Q}$ and the coordinates of a point on the chief ray, the coordinates $(x, y, z)$ of the point of incidence of the ray on the toric must be determined (transfer process); next $\boldsymbol{U}$ at the point of incidence must be found and $\boldsymbol{Q}^{\prime}$; the direction of the refracted chief ray, determined (refraction process). The quantities $c_{r}, c_{s}, \boldsymbol{R}$, and $\boldsymbol{S}$ will be known for the incident pencil and $c_{v}, c_{v}, \boldsymbol{V}$, and $\boldsymbol{W}$ can be found, since the point of incidence is known; from these the quantities $c_{r}^{\prime}, c_{s}^{\prime}, \boldsymbol{R}^{\prime}$, and $\boldsymbol{S}^{\prime}$ for the refracted pencil must be determined. This last, the astigmatism calculation, will be described in section 5 .

\subsection{Transfer Process}

The transfer problem is that of finding the intersection of a straight line with a toric. A direct approach would require the solution of an algebraic equation of the fourth degree; this would be a laborious process, so an iterative method was adopted. In addition to the general advantage of iterative methods, that mistakes in the early stages of the calculation are automatically eliminated at the cost of extra computing time, this method also selects the correct solution from the four possible solutions. 
Let $P_{1}$ be a point on the ray near the required point of incidence. ${ }^{8}$ A point $P_{1}^{\prime}$ on the toric is found with the same $y$ and $z$ coordinates and the tangent plane $T_{1}$ at $P_{1}^{\prime}$ is determined. The next approximation $P_{2}$ is taken at the intersection of the ray with $T_{1}$ and the cycle $P_{2} \rightarrow P_{2}^{\prime} \rightarrow T_{2} \rightarrow P_{3} \ldots$. repeated until the coordinates of $P_{n}$ and $P_{n+1}$ are the same to the required number of significant figures. These are then the coordinates of $P$, the point of incidence.

Let the coordinates of $P_{n}$ be $\left(x_{n}, y_{n}, z_{n}\right)$ and those of $P_{n}^{\prime}$ be $\left(x_{n}^{\prime}, y_{n}, z_{n}\right)$; then $x_{n}^{\prime}$ is obtained by substituting $y_{n}$ and $z_{n}$ in eq (6). The equation of the plane $T_{n}$ is $\lambda_{n}\left(x-x_{n}^{\prime}\right)+\mu_{n}\left(y-y_{n}\right)+$ $\nu_{n}\left(z-z_{n}\right)=0$,

where $\lambda_{n}, \mu_{n}$, and $\nu z$ are given by eq $(7)$ so that $x_{n+1}$ is given by

$$
\left.\begin{array}{rl}
x_{n+1} & =x_{n}+\delta_{n} \\
\delta_{n} & =\frac{\lambda_{n} X\left(x_{n}^{\prime}-x_{n}\right)}{\lambda_{n} X+\mu_{n} Y+\nu_{n} Z}
\end{array}\right\} .
$$

Also,

$$
\left.\begin{array}{l}
y_{n+1}=y_{n}+\frac{Y}{X} \delta_{n} \\
z_{n+1}=z_{n}+\frac{Z}{X} \delta_{n}
\end{array}\right\}
$$

This completes the iterative cycle. It can be shown to be a second-order iteration, ${ }^{9}$ that is, $\delta_{n+1}$ is of order of magnitude $\delta_{n}^{2}$, so that it is always rapidly convergent, provided the initial approximation is good enough.

The choice of the initial approximation depends on the available means of computation. If a high-speed automatic-computing engine is available, $P_{1}$ may be taken as the point where the ray meets the $y z$ plane. Let $\left(x_{0}, y_{0}, z_{0}\right)$ be the coordinates of the point $P_{0}$ where the ray leaves the previous refracting surface (or the entrance pupil); ${ }^{10}$ then $P_{1}$ is given by

$$
\left.\begin{array}{l}
x_{1}=0, \\
y_{1}=y_{0}-x_{0} Y / X \\
z_{1}=z_{0}-x_{0} Z / X
\end{array}\right\}
$$

Starting from this approximation, some four or five iterations would probably be needed to reach a result correct to six significant figures.

If a desk computing machine is to be used, an appreciable saving in computing time can be effected by using an improved first estimate. It usually happens that chief rays directed at the same pupil have already been traced in the meridian and equatorial sections of the toric. Let the $x$ coordinates of their points of incidence be $x_{y}$ and $x_{z}$, respectively. The section of the toric by a plane through the $x$ axis and making an angle $\alpha$ with the $x y$ plane is approximately a circle, of curvature $c_{y} \cos ^{2} \alpha+c_{x} \sin ^{2} \alpha$, so that a chief ray in this plane will meet the toric at a point of which the $x$ coordinate is approximately $x_{y} \cos ^{2} \alpha+x_{z} \sin ^{2} \alpha$. This $P_{1}$ is given by

$$
\left.\begin{array}{l}
x_{1}=x_{y} \cos ^{2} \alpha+x_{z} \sin ^{2} \alpha \\
y_{1}=y_{0}+\left(x_{1}-x_{0}\right) Y / X \\
z_{1}=z_{0}+\left(x_{1}-x_{0}\right) Z / X
\end{array}\right\}
$$

This approximation is usually so good that only one iteration is needed to obtain six significant figures in the coordinates of $P$.

8 The method of obtaining $P_{1}$ will be discussed below.

${ }^{9}$ W. Weinstein, Proc. Phys. Soc. (London) [B] 65, 731 (1952).

${ }^{10}\left(x_{0}, y_{0}, z_{0}\right)$ are taken in the coordinate system referring to the current surface, not that on which $P_{0}$ lies. 


\subsection{Refraction Process}

The refraction calculation for the chief ray can now be carried out by well-known formulas. Let $I$ be the angle of incidence and $n$ the index of refraction of the medium in which the ray is incident; let primes denote corresponding refracted quantities. Then

$$
\cos I=\boldsymbol{Q} \cdot \boldsymbol{U}
$$

$$
\begin{aligned}
& \cos I^{\prime}=+\left(1-\frac{n^{2}}{n^{\prime 2}}\left(1-\cos ^{2} I\right)\right)^{1 / 2}, \\
& \boldsymbol{Q}^{\prime}=\frac{n}{n^{\prime}} \boldsymbol{Q}+\left(\cos I^{\prime}-\frac{n}{n^{\prime}} \cos I\right) \boldsymbol{U} .
\end{aligned}
$$

In eq (14) $\boldsymbol{Q} \cdot \boldsymbol{U}$ denotes the scalar product of $\boldsymbol{Q}$ and $\boldsymbol{U}$.

This completes the refraction process and the coordinates and direction cosines of the refracted ray are then used, with a suitable change of origin, for the incident ray at the next surface.

\section{Astigmatism Calculation}

In order to determine equations giving $\boldsymbol{R}^{\prime}, \boldsymbol{S}^{\prime}, c_{r}^{\prime}$, and $c_{s}^{\prime}$, in terms of known quantities, a general theorem is required. Referring to figure 4, let $P$ be the point of incidence of the chief ray, and let $B$ be the point of incidence of another ray of the pencil. Suppose the incident and refracted wavefronts through $P$ to be constructed, and let the ray through $B$ meet these in $C$ and $C^{\prime}$, respectively. ${ }^{11}$ Then, since the optical path length between two wavefronts is the same along any ray, it follows that $n \overline{C B}=n^{\prime} \overline{C^{\prime} B^{\prime}}$ or, if $\overline{C B}$ is denoted by $q$,

$$
\Delta(n q)=0 .
$$

Here the symbol, $\Delta$, means that the refracted quantity minus the incident quantity is to be taken. This relationship is true for any relative positions of $P$ and $B$ and for any shape of refracting surface.

In order to use eq (17) for the present purpose, $q$ and $q^{\prime}$ will be expressed in terms of the coordinates of the point $B$ on the refracting surface and the parameters of the wavefronts. The resulting equation will give the normal curvature of the refracted wavefront in a section containing the ray through $B$ and by differentiating with respect to the azimuth of $B$ the maximum and minimum normal curvatures, $c_{r}^{\prime}$ and $c_{s}^{\prime}$, can be found. The same calculation gives the directions $\boldsymbol{R}^{\prime}$ and $\boldsymbol{S}^{\prime}$ of the principal sections.

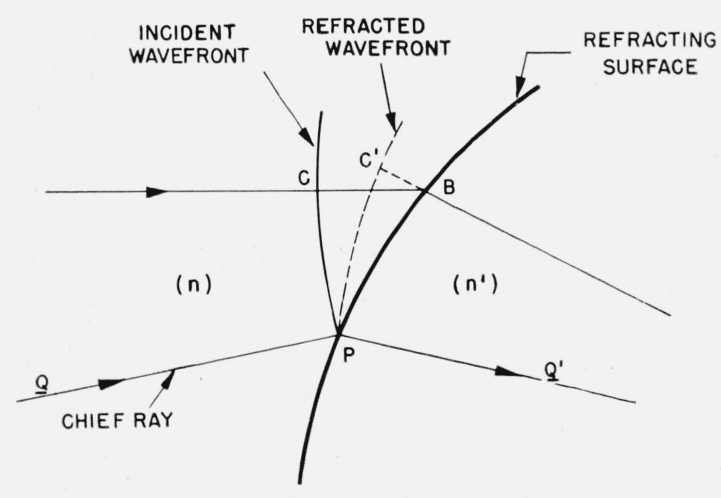

Figure 4.

11 The part of the refracted wavefront shown in the diagram is virtual. 


\subsection{Normal Curvature of the Refracted Wavefront}

Figure 5 shows the refracting surface near the point of incidence. Let $P$ and $B$ have the same significance as in figure 4 and let $\boldsymbol{V}$ and $\boldsymbol{W}$ be the unit vectors along the principal directions. Let $B_{1}$ be the projection of $B$ on the $\boldsymbol{V} \boldsymbol{W}$ plane; let $\alpha$ be the angle between $P B_{1}$ and $\boldsymbol{V}$, and let $P B$ be denoted by $p$. Then the position of $B$ relative to $P$ will be given by $p$ and $\alpha$ as coordinates.

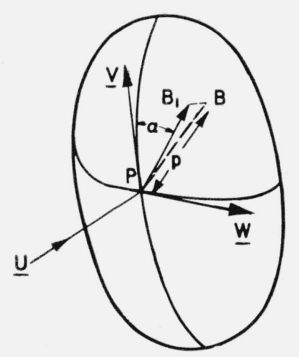

FiguRE 5 .

In order to express $q$ in terms of $p$ and $\alpha$, use is made of the fact that $C B$ in figure 4 is a normal to the wavefront. It is found (see section 5.1, a) that

$$
\begin{gathered}
q=p(\boldsymbol{Q} \cdot \boldsymbol{V} \cos \alpha+\boldsymbol{Q} \cdot \boldsymbol{W} \sin \alpha)+\frac{1}{2} p^{2}\left\{\boldsymbol{Q} \cdot \boldsymbol{U}\left(c_{v} \cos ^{2} \alpha+c_{w} \sin ^{2} \alpha\right)-c_{r}(\boldsymbol{R} \cdot \boldsymbol{V} \cos \alpha+\boldsymbol{R} \cdot \boldsymbol{W} \sin \alpha)^{2}\right. \\
\left.-c_{s}(\boldsymbol{S} \cdot \boldsymbol{V} \cos \alpha+\boldsymbol{S} \cdot \boldsymbol{W} \sin \alpha)^{2}\right\}+ \text { terms in } p^{3},
\end{gathered}
$$

and there is a similar equation for $q^{\prime}$.

Substituting for $q$ and $q^{\prime}$ in eq (17),

$$
\begin{gathered}
p \Delta n(\boldsymbol{Q} \cdot \boldsymbol{V} \cos \alpha+\boldsymbol{Q} \cdot \boldsymbol{W} \sin \alpha)+\frac{1}{2} p^{2} \Delta n\left\{\boldsymbol{Q} \cdot \boldsymbol{U}\left(c_{v} \cos ^{2} \alpha+c_{w} \sin ^{2} \alpha\right)-c_{r}(\boldsymbol{R} \cdot \boldsymbol{V} \cos \alpha+\boldsymbol{R} \cdot \boldsymbol{W} \sin \alpha)^{2}\right. \\
\left.-c_{s}(\boldsymbol{S} \cdot \boldsymbol{V} \cos \alpha+\boldsymbol{S} \cdot \boldsymbol{W} \sin \alpha)^{2}\right\}+ \text { terms in } p^{3}=0 .
\end{gathered}
$$

Only terms up to and including the square of $p$ are taken since, as explained in section 2, this degree of approximation takes account of astigmatism.

Equation 19 holds good for any values of $p$ and $\alpha$, so that the coefficients of different powers of $p$ may be separately equated to zero. If this is done, the linear term yields an alternative form of Snell's law and on putting $\alpha=0$ and $\pi / 2$, the equations

$$
\Delta[n \boldsymbol{Q} \cdot \boldsymbol{V}]=\Delta[n \boldsymbol{Q} \cdot \boldsymbol{W}]=0
$$

are obtained. These could serve as a check on the refraction calculation.

Equating the coefficient of $p^{2}$ in eq (19) to zero and dividing through by $1-\left(\boldsymbol{Q}^{\prime} \cdot \boldsymbol{V} \cos \alpha+\right.$ $\left.\boldsymbol{Q}^{\prime} \cdot \boldsymbol{W} \sin \alpha\right)^{2}$ gives

$$
\begin{aligned}
& \frac{c_{r}^{\prime}\left(\boldsymbol{R}^{\prime} \cdot \boldsymbol{V} \cos \alpha+\boldsymbol{R}^{\prime} \cdot \boldsymbol{W} \sin \alpha\right)^{2}+c_{s}^{\prime}\left(\boldsymbol{S}^{\prime} \cdot \boldsymbol{V} \cos \alpha+\boldsymbol{S}^{\prime} \cdot \boldsymbol{W} \sin \alpha\right)^{2}}{1-\left(\boldsymbol{Q}^{\prime} \cdot \boldsymbol{V} \cos \alpha+\boldsymbol{Q}^{\prime} \cdot \boldsymbol{W} \sin \alpha\right)^{2}} \\
& =\frac{n c_{r}(\boldsymbol{R} \cdot \boldsymbol{V} \cos \alpha+\boldsymbol{R} \cdot \boldsymbol{W} \sin \alpha)^{2}+n c_{s}(\boldsymbol{S} \cdot \boldsymbol{V} \cos \alpha+\boldsymbol{S} \cdot \boldsymbol{W} \sin \alpha)^{2}+\left(c_{v} \cos ^{2} \alpha+c_{w} \sin \alpha\right) \Delta n \boldsymbol{Q} \cdot \boldsymbol{U}}{n^{\prime}\left[1-\left(\boldsymbol{Q}^{\prime} \cdot \boldsymbol{V} \cos \alpha+\boldsymbol{Q}^{\prime} \cdot \boldsymbol{W} \sin \alpha\right)^{2}\right]} .
\end{aligned}
$$

Now the equation of the refracted wavefront can be shown to be

$$
x=\frac{\left[c_{r}^{\prime}\left(\boldsymbol{R}^{\prime} \cdot \boldsymbol{V} \cos \alpha+\boldsymbol{R}^{\prime} \cdot \boldsymbol{W} \sin \alpha\right)^{2}+c_{s}^{\prime}\left(\boldsymbol{S}^{\prime} \cdot \boldsymbol{V} \cos \alpha+\boldsymbol{S}^{\prime} \cdot \boldsymbol{W} \sin \alpha\right)^{2}\right]\left(x^{2}+y^{2}\right),}{\left(\boldsymbol{R}^{\prime} \cdot \boldsymbol{V} \cos \alpha+\boldsymbol{R}^{\prime} \cdot \boldsymbol{W} \sin \alpha\right)^{2}+\left(\boldsymbol{S}^{\prime} \cdot \boldsymbol{V} \cos \alpha+\boldsymbol{S}^{\prime} \cdot \boldsymbol{W} \sin \alpha\right)^{2}},
$$

taking the $x, y$, and $z$ axes along $\boldsymbol{Q}^{\prime}, \boldsymbol{R}^{\prime}$, and $\boldsymbol{S}^{\prime}$. Thus the left hand side of eq $(21)$ is $c^{\prime}(\alpha)$, the 
normal curvature of the refracted wavefront in a section containing the point $B$ with angular coordinate $\alpha$ on the toric.

$$
\text { a. Derivation of Equation (18) }
$$

Two auxiliary coordinate systems are required, each with origin at $P$, the point of incidence. One, the $x, y, z$ system, has its axes respectively parallel to $\boldsymbol{Q}, \boldsymbol{R}$, and $\boldsymbol{S}$; the other, $x^{\prime}, y^{\prime}$, and $z^{\prime}$ has its axes parallel to $\boldsymbol{U}, \boldsymbol{V}$, and $\boldsymbol{W}$. The two are related by the equations

$$
\begin{array}{r}
x=(\boldsymbol{Q} \cdot \boldsymbol{U}) x^{\prime}+(\boldsymbol{Q} \cdot \boldsymbol{V}) y^{\prime}+(\boldsymbol{Q} \cdot \boldsymbol{W}) z^{\prime}, \quad y=(\boldsymbol{R} \cdot \boldsymbol{U}) x^{\prime}+(\boldsymbol{R} \cdot \boldsymbol{V}) y^{\prime}+(\boldsymbol{R} \cdot \boldsymbol{W}) z^{\prime}, \\
z=(\boldsymbol{S} \cdot \boldsymbol{U}) x^{\prime}+(\boldsymbol{S} \cdot \boldsymbol{V}) y^{\prime}+(\boldsymbol{S} \cdot \boldsymbol{W}) z^{\prime} .
\end{array}
$$

If the point $B$ on the refracting surface (fig. 4) has polar coordinates $(p, \alpha)$, its coordinates in the $x^{\prime}, y^{\prime}, z^{\prime}$ system are ${ }^{12}$

$$
x^{\prime}=\frac{1}{2}\left(c_{v} \cos ^{2} \alpha+c_{w} \sin ^{2} \alpha\right) p^{2}+O\left(p^{4}\right), \quad y^{\prime}=p \cos \alpha+O\left(p^{3}\right), \quad z^{\prime}=p \sin \alpha+O\left(p^{3}\right) .
$$

Let $B$ have coordinates $(u, v, w)$ in the $(x, y, z)$ system; then

$$
\begin{aligned}
u & =\frac{1}{2}(\boldsymbol{Q} \cdot \boldsymbol{U})\left(c_{v} \cos ^{2} \alpha+c_{w} \sin ^{2} \alpha\right) p^{2}+(\boldsymbol{Q} \cdot \boldsymbol{V}) p \cos \alpha+(\boldsymbol{Q} \cdot \boldsymbol{W}) p \sin \alpha+O\left(p^{3}\right), \\
v & =(\boldsymbol{R} \cdot \boldsymbol{V}) p \cos \alpha+(\boldsymbol{R} \cdot \boldsymbol{W}) p \sin \alpha+O\left(p^{2}\right), \\
w & =(\boldsymbol{S} \cdot \boldsymbol{V}) p \cos \alpha+(\boldsymbol{S} \cdot \boldsymbol{W}) p \sin \alpha+O\left(p^{2}\right) .
\end{aligned}
$$

Let $(x, y, z)$ be the coordinates of $C$ in the $x, y, z$ system, so that

$$
x=\frac{1}{2}\left(c_{r} y^{2}+c_{s} z^{2}\right)+O\left(y^{3}, z^{3}\right) .
$$

The direction cosines of the normal to the wavefront at $C$ are, to the approximation $O\left(y^{2}, z^{2}\right)$, $\left(1,-c_{r} y,-c_{s} z\right)$, and since $C B$ is a ray, or wavefront normal, it follows that

$$
u=x+q, \quad v=y-c_{r} y q, \quad w=z-c_{s} z q .
$$

There are now enough relations to eliminate $x, y, z, u, v$, and $w$. The resulting equation involving $p, q$, and $\alpha$ can be solved for $q$, remembering that terms which are $O\left(p^{3}, q^{3}\right)$ can be neglected, since only astigmatism is in question. The solution for $q$ is found to be eq (18).

\subsection{The Principal Curvatures of the Refracted Wavefront}

The principal curvatures $c_{s}^{\prime}$ and $c_{r}^{\prime}$ are the maximum and minimum values of $c^{\prime}(\alpha)$. Since all quantities on the right of eq (21) are known with the exception of $\alpha, c_{r}^{\prime}$ and $c_{s}^{\prime}$ can be obtained in the usual way by differentiating with respect to $\alpha$ and equating to zero. Let

$$
\begin{aligned}
& a=\frac{1}{2}\left[n c_{r}\left((\boldsymbol{R} \cdot \boldsymbol{V})^{2}+(\boldsymbol{R} \cdot \boldsymbol{W})^{2}\right)+n c_{s}\left((\boldsymbol{S} \cdot \boldsymbol{V})^{2}+(\boldsymbol{S} \cdot \boldsymbol{W})^{2}\right)+\left(c_{v}+c_{w}\right) \Delta n \boldsymbol{Q} \cdot \boldsymbol{U}\right] \\
& b=\frac{1}{2}\left[n c_{r}\left((\boldsymbol{R} \cdot \boldsymbol{V})^{2}-(\boldsymbol{R} \cdot \boldsymbol{W})^{2}\right)+n c_{s}\left((\boldsymbol{S} \cdot \boldsymbol{V})^{2}-(\boldsymbol{S} \cdot \boldsymbol{W})^{2}\right)+\left(c_{v}+c_{w}\right) \Delta n \boldsymbol{Q} \cdot \boldsymbol{U}\right], \\
& c=n c_{r}(\boldsymbol{R} \cdot \boldsymbol{V})(\boldsymbol{R} \cdot \boldsymbol{W})+n c_{s}(\boldsymbol{S} \cdot \boldsymbol{V})(\boldsymbol{S} \cdot \boldsymbol{W}), \\
& d=\frac{1}{2}\left(\left(2-\left(\boldsymbol{Q}^{\prime} \cdot \boldsymbol{V}\right)^{2}-\left(\boldsymbol{Q}^{\prime} \cdot \boldsymbol{W}\right)^{2}\right),\right. \\
& e=\frac{1}{2}\left(\left(\boldsymbol{Q}^{\prime} \cdot \boldsymbol{V}\right)^{2}-\left(\boldsymbol{Q}^{\prime} \cdot \boldsymbol{W}\right)^{2}\right), \\
& f=-\left(\boldsymbol{Q}^{\prime} \cdot \boldsymbol{V}\right)\left(\boldsymbol{Q}^{\prime} \cdot \boldsymbol{W}\right) .
\end{aligned}
$$

The equation for $\alpha$ is

$$
(e a-b d) \sin ^{2} \alpha+(d c-a f) \cos ^{2} \alpha=(b f-e c),
$$

\footnotetext{
12 The expression $O\left(p^{n}\right)$ used in this section denotes terms of order of magnitude $p^{n}$.
} 
and the two solutions are

where ${ }^{13}$

$$
\alpha_{1}=\frac{\gamma-\beta}{2}, \quad \alpha_{2}=\frac{\pi}{2}-\frac{\gamma+\beta}{2}
$$

and

$$
\beta=\arcsin \frac{d c-a f}{+\left[(e a-b d)^{2}+(d c-a f)^{2}\right]^{1 / 2}}
$$

$$
\gamma=\arcsin \frac{b f-e c}{\left[(e a-b d)^{2}+(d c-a f)^{2}\right]^{1 / 2}}
$$

The values of $c_{s}^{\prime}$ and $c_{r}^{\prime}$ are then obtained by putting $\alpha=\alpha_{1}$ and $\alpha_{2}$ in the right hand side of eq $(21)$.

\subsection{Principal Directions on the Refracted Wavefront}

In figure 6 , let $P$ be the point of incidence, $P B$ the refracting surface, and $P C^{\prime}$ the refracted wavefront. Let $\boldsymbol{Q}^{\prime}$ be the unit vector along the chief ray and let $C^{\prime} B$ be another ray of the astigmatic pencil. Let the projections of $C^{\prime}$ and $B$ on the tangent planes to the wavefront and refracting surface be $C_{1}^{\prime}$ and $B_{1}$ respectively. Then $\boldsymbol{V} \cos \alpha+\boldsymbol{W} \sin \alpha$ is a vector in the direction $P B_{1}$, so that a unit vector in the direction $P C_{1}{ }^{\prime}$ is given by

$$
\frac{\boldsymbol{V} \cos \alpha+\boldsymbol{W} \sin \alpha-\boldsymbol{Q}^{\prime}\left(\boldsymbol{Q}^{\prime} \cdot \boldsymbol{V} \cos \alpha+\boldsymbol{Q}^{\prime} \cdot \boldsymbol{W} \sin \alpha\right)}{\left[1-\left(\boldsymbol{Q}^{\prime} \cdot \boldsymbol{V} \cos \alpha+\boldsymbol{Q}^{\prime} \cdot \boldsymbol{W} \sin \alpha\right)^{2}\right]^{1 / 2}} .
$$

Thus, the vectors $\boldsymbol{R}^{\prime}$ and $\boldsymbol{S}^{\prime}$ are obtained by putting $\alpha=\alpha_{1}$ and $\alpha_{2}$ in the expression (31). It should be noticed that in this derivation it was assumed that the points $C^{\prime}$ and $B$ lie on a plane through $\boldsymbol{Q}^{\prime}$, i. e., that the ray through $C^{\prime}$ intersects the chief ray. This is not strictly true in general, but the error depends on terms in the equation of the wavefront of the third degree in the aperture, so that astigmatism calculations are not affected.

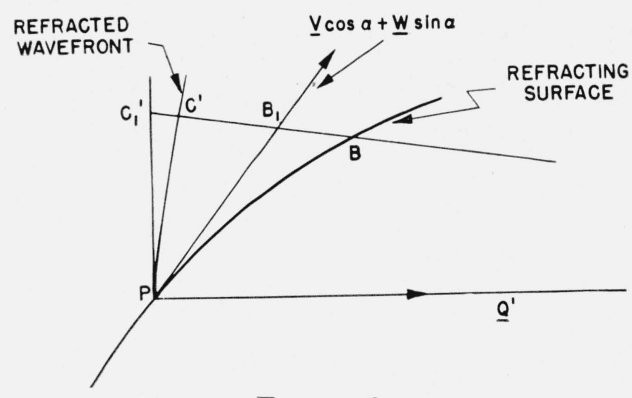

Figure 6.

This completes the refraction computation for astigmatism. The $\boldsymbol{R}^{\prime}$ and $\boldsymbol{S}^{\prime}$ vectors are transferred without change to be the new $\boldsymbol{R}$ and $\boldsymbol{S}$ vectors at the next surface. In order to transfer the curvatures the distance $L$ between surfaces along the chief ray is required; this is easily found to be $\left(x-x_{0}\right) / X$, in the notation of section 4.1. Then the new curvatures are given by

$$
\left.\begin{array}{l}
\left(1 / c_{r}\right)_{+1}=\left(1 / c_{r}\right)^{\prime}-L \\
\left(1 / c_{s}\right)_{+1}=\left(1 / c_{s}\right)^{\prime}-L
\end{array}\right\}
$$

${ }^{13}$ Of the possible solutions for $\beta$, one must be chosen which satisfies

$$
\beta=\arccos \frac{e a-b d}{+\left[(e a-b d)^{2}+(d c-a f)^{2}\right]^{1 / 2}}+\text { a multiple of } 2 \pi
$$




\section{Input Data}

If the wavefront incident on the first surface is not already astigmatic, some arbitrary directions for $\boldsymbol{R}$ and $\boldsymbol{S}$ must be assigned. Similarly if a refracting surface is spherical, directions for $\boldsymbol{V}$ and $\boldsymbol{W}$ must be assigned.

These pairs of vectors must form trirectangular sets with $\boldsymbol{Q}$ and $\boldsymbol{U}$, respectively, but subject to this condition they can be chosen to suit computational convenience. Choosing $\boldsymbol{R}$ to be coplanar with $\boldsymbol{Q}$ and the $y$ axis, it is found that

$$
\left.\begin{array}{l}
\boldsymbol{R}=\frac{\left(-X Y, 1-Y^{2},-Y Z\right)}{\left(1-Y^{2}\right)^{1 / 2}} \\
\boldsymbol{S}=\frac{(Z, 0,-X)}{\left(1-Y^{2}\right)^{1 / 2}}
\end{array}\right\}
$$

Since $Y^{2}$ is rarely greater than 0.1 , the vectors so defined are well determined numerically. In a similar way $\boldsymbol{V}$ and $\boldsymbol{W}$ can be taken as

$$
\left.\begin{array}{c}
\boldsymbol{V}=\frac{\left(-\lambda \mu, 1-\mu^{2},-\mu \nu\right)}{\left(1-\mu^{2}\right)^{1 / 2}} \\
\boldsymbol{W}=\frac{(\nu, 0,-\lambda)}{\left(1-\mu^{2}\right)^{1 / 2}}
\end{array}\right\} .
$$

It is also necessary to choose between ring and barrel shapes for the toric surfaces. In the equations of section 3.1 the rotation axis of the toric is taken parallel to the $y$ axis and the toric will be ring or barrel shape according as $c_{y}$ is numerically geater or less than $c_{z}$. Thus, if it is required to have a barrel shape with $\left|c_{y}\right|>\left|c_{z}\right|$ or a ring shape with $\left|c_{y}\right|<\left|c_{z}\right|$, the equations must be changed so that the rotation axis is parallel to the $z$ axis. This can be done by interchanging $y$ and $z$ in the symbols and subscripts of eq (4) to (8), inclusive, as mentioned in section 3.1.

\section{Presentation of Results}

\subsection{A Numerical Example}

In the application to spectacle lenses the results are presented in the form of a diagram giving the astigmatism of a number of pencils and the directions of the principal sections on the wave front. An example is given in figure 7 .
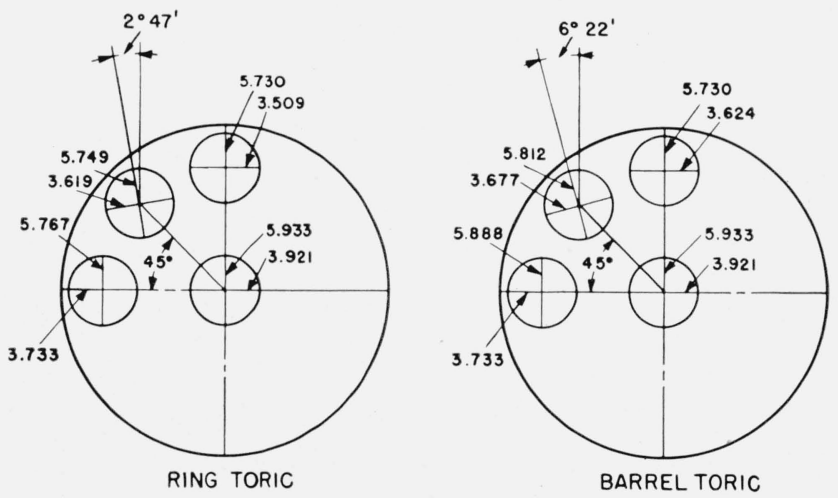

Figure 7. 
The wavefront curvatures are marked on the appropriate sections, in diopters with respect to the vertex sphere. The apparent rotation of the principal sections of the wavefront can be found from the consideration that the vertical plane containing the chief ray contains the direction which is apparently vertical to the eye. This plane is the plane through $\boldsymbol{Q}$ and parallel to the $y$ axis. From this it can be shown that the angle, $A$, through which the $\boldsymbol{R}$ and $\boldsymbol{S}$ vectors appear to be turned is given by

$$
\tan A=\frac{r_{2}}{s_{2}}
$$

where $r_{2}$ and $s_{2}$ are the $y$ components of $\boldsymbol{R}$ and $\boldsymbol{S}$.

The nominal powers of the lens in this example were 5.5 diopters in the vertical section and 3.62 diopters in the horizontal section. The curvatures of the front (toric) surface were $+0.2199 \mathrm{~cm}^{-1}$ and $+0.1839 \mathrm{~cm}^{-1}$, that of the back (spherical) surface was $-0.1147 \mathrm{~cm}^{-1}$, the center thickness was $0.48 \mathrm{~cm}$ and the refractive index was taken as 1.523 . The chief ray was made to intersect the axis $27 \mathrm{~mm}$ behind the lens at an angle of $30^{\circ}$. The results for both ring and barrel shapes with the same nominal curvatures are given.

Computations have also been done on the prescriptions of a number of lenses and measurements have been made on these with a vertex power measuring instrument. The agreement is satisfactory within the limits of experimental error.

\subsection{Discussion}

In the numerical example given above the rotation of the focal lines in intermediate azimuths is negligible; this may be expected to be the case for all but very heavily cylindrical spectacle lenses of best-form type. It can also be seen that the variation of astigmatism over the field is less for the barrel than for the ring shape $(0.14 \mathrm{D}$ and $0.21 \mathrm{D}$, respectively). This, of course, is not a general conclusion, but it suggests that further computation would be desirable. It is possible that for heavy cylinders a considerable improvement might be obtained by using barrel shapes, but this might be outweighed by the increased manufacturing costs. This would depend also on visual tolerances for astigmatism; it seems that more experimental work is required on this subject.

A different question is that of the accuracy with which torics are produced. The principal curvatures check within the limits of accuracy of a lens gage, and the sagittal curvatures change or remain constant along the meridan or equatorial sections, respectively, to the same accuracy. However, this may not be enough to prove that the lens surface is a true toric to an accuracy which justifies the calculations for chief rays in intermediate azimuths. The mode of manufacture is certainly such as to produce a very close approximation to a toric if the surfacing machine really acts in the intended manner, but it is quite possible that distortions and nonuniform wear in the tools and machine parts may cause departures from the toric shape. No measurements other than checks on the principal curvatures seem to have been made. A detailed check on the $x$ coordinates of a network of points would be needed.

Thanks are due to my friends and colleagues of the Optical Instruments Section for their interest in this work, in particular O. N. Stavroudis and Loyd Sutton; the latter did the numerical computations and checked the mathematics.

Washington, June 29, 1954. 